\title{
Vigilancia de la infección por Rickettsia sp. en capibaras (Hydrochoerus hydrochaeris) un modelo potencial de alerta epidemiológica en zonas endémicas
}

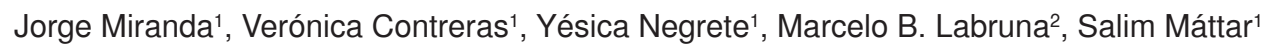 \\ 1 Instituto de Investigaciones Biológicas del Trópico, Universidad de Córdoba, Montería, Colombia \\ 2 Departamento de Medicina Veterinária Preventiva e Saúde Animal, Faculdade de Medicina Veterinária e \\ Zootecnia, Universidade de São Paulo, São Paulo, Brasil
}

Introducción. Los capibaras o chigüiros (Hydrochoerus hydrochaeris) son huéspedes amplificadores de Rickettsia sp. Usualmente se encuentran parasitados por la garrapata Amblyomma cajennense, principal vector de rickettsiosis en Suramérica. Los capibaras pueden ser usados como potenciales centinelas de la circulación de rickettsias.

Objetivo. Detectar anticuerpos contra Rickettsia sp. del grupo de las fiebres manchadas en capibaras de una zona rural del municipio de Montería, departamento de Córdoba.

Material y métodos. Se analizaron 36 sueros de capibaras de una zona rural de Montería (vereda San Jerónimo) en Córdoba. Para la detección de anticuerpos lgG se practicó inmunofluorescencia indirecta, que utilizó antígenos de la cepa Taiaçu de Rickettsia rickettsii de Brasil. Los sueros de los capibaras fueron diluidos 1:64. Se capturaron las garrapatas que se encontraban parasitando los capibaras y se clasificaron hasta su especie.

Resultados. La seroprevalencia contra Rickettsia sp. del grupo de la fiebres manchadas encontrada fue de $22 \%$ (8 capibaras); se encontraron cuatro sueros con título de 1:64, tres sueros con título $1: 128$ y un suero presentó titulación de 1:512. Todas las garrapatas $(n=933)$ fueron identificadas taxonómicamente como $A$. cajennense.

Conclusión. En Colombia existen zonas endémicas de rickettsiosis y la aparición de brotes anuales lo confirma (Necoclí, 2006; Los Córdobas, 2007, y Altos de Mulatos, 2008). El presente estudio reporta por primera vez la presencia de infección natural por rickettsia del grupo de las fiebres manchadas en capibaras de Colombia. Los hallazgos sugieren que los capibaras pueden ser usados como potenciales centinelas de la circulación de rickettsias y marcadores de las áreas de riesgo para la transmisión de rickettsiosis.

Palabras clave: Rickettsia sp., infecciones por rickettsiaceae, roedores, garrapatas, técnica indirecta del anticuerpo fluorescente, Colombia.

Surveillance of Rickettsia sp. infection in capybaras (Hydrochoerus hydrochaeris) a potential model of epidemiological alert in endemic areas

Introduction. Capybaras (Hydrochoerus hydrochaeris) are considered amplifying hosts of Rickettsia $\mathrm{sp}$. These rodents are usually parasitized by the tick vector, Amblyomma cajennense, the main vector of rickettsioses in humans and animals in South America. Capybaras can be used as sentinels in detection of circulation of rickettsiae.

Objective. Antibodies to rickettsiae of spotted fever group were detected in capybaras in a rural area of Cordoba Province, northern Colombia.

Materials and methods. Sera were analyzed from 36 capybaras in a rural area of Monteria (village of San Jeronimo) in Córdoba. For the detection of $\mathrm{lgG}$ antibodies, indirect immunofluorescence was performed. The antigens were derived from $R$. rickettsia strain Taiaçu isolated in Brazil. Capybara sera were diluted 1:64 for IFA analysis. Ticks were collected from each capybara (also known as chigüiro) and identified to species.

Results. The seroprevalence of spotted fever group Rickettsia was $22 \%$ (8 capybaras). Four sera had a titer of 1:64, 3 had a titer of 1:128 and one serum had a titer of 1:512. All ticks removed from the capybaras ( $n=933$ ) were taxonomically identified as Amblyomma cajennense.

Conclusion. Colombia has areas endemic for rickettsioses, as indicated by confirmed annual outbreaks. The current study reports the first evidence of natural rickettsial infection of the spotted fever group in capybaras from Colombia. The findings suggest that capybaras can be used as sentinels for the circulation of rickettsiae and can identify endemic areas for the transmission of rickettsial diseases.

Key words: Rickettsia sp., rickettsiaceae infections, rodentia, ticks; fluorescent antibody technique, indirect; Colombia. 
Las rickettsiosis son enfermedades zoonóticas febriles agudas causadas por bacterias Gram negativas intracelulares obligadas, de la familia Rickettsiaceae. Varios artrópodos, incluyendo garrapatas, pulgas y piojos, pueden actuar como vectores y reservorios (1). Las especies del género Rickettsia se dividen actualmente en cuatro grupos: grupo del tifus, compuesto por Rickettsia prowazekii y $R$. typhi, asociado a piojos y pulgas; grupo de las fiebres manchadas que incluye más de 20 especies transmitidas por garrapatas, entre otras $R$. rickettsii, $R$. conorii, $R$. sibirica y $R$. japonica; grupo ancestral que incluye las especies $R$. bellii y $R$. canadensis, asociadas a garrapatas; y el grupo transicional que incluye las especies $R$. akari, $R$. felis y $R$. australis, asociadas a piojos, pulgas y garrapatas, respectivamente $(2,3)$.

Hasta el año 2000 sólo tres especies de Rickettsia habían sido descritas en Suramérica: $R$. rickettsii, $R$. prowazekii y $R$. typhi. En los últimos años, se han reportado siete especies diferentes de Rickettsia: $R$. felis aisladas de pulgas infectadas en Argentina, Brasil, Chile, Perú y Uruguay; $R$. parkeri, de garrapatas en Uruguay, Argentina y Brasil; R. massiliae, de garrapatas infectadas en Argentina; Candidatus " $R$. amblyommii", de garrapatas infectadas de Argentina, Brasil y la Guyana Francesa; $R$. bellii, que infecta garrapatas en Argentina y Brasil; $R$. rhipicephali, que infecta garrapatas en Brasil; Candidatus " $R$. andeanae", de garrapatas en Perú y Argentina; y Rickettsia sp. Argentina, que infecta garrapatas en Argentina. Todas estas especies están clasificadas dentro del grupo de las fiebres manchadas, excepto $R$. bellii que pertenece al grupo ancestral (4-6).

Generalmente, los síntomas clínicos de las rickettsiosis de grupo de las fiebres manchadas comienzan de forma aguda entre seis y diez días después de la picadura del vector, con fiebre, cefalea intensa, mialgia y erupción macular o máculo-papular y linfadenopatía local (excepto $R$. rickettsii). Los principales signos clínicos varían de leves a graves y fatales, dependiendo de la especie de rickettsia involucrada (7). $R$. rickettsii es el agente etiológico de la fiebre manchada de las Montañas Rocosas (Rocky Mountain spotted

Correspondencia

Salim Máttar, Instituto de Investigaciones Biológicas del Trópico, Universidad de Córdoba, Montería, Colombia

Teléfono: 756 0710; fax: 7560710

mattarsalim@hotmail.com

Recibido: 13/08/10; aceptado:22/02/11 fever); ésta es la rickettsia más patógena para humanos y algunos animales. En América se ha reportado en Canadá, Estados Unidos, México, Costa Rica, Panamá, Colombia, Brasil y Argentina. En Brasil se conoce como fiebre maculosa del Brasil y, en Colombia, como fiebre de Tobia (8).

En Centroamérica y Suramérica, R. rickettsii es transmitida a los humanos principalmente por Amblyomma cajennense (4). Sin embargo, las tasas de infección por $R$. rickettsii en las garrapatas son bajas en condiciones naturales (8-10). En el caso de $A$. cajennense, puede atribuirse principalmente a que esta garrapata es menos propensa a la infección por $R$. rickettsii que otras especies (11). El papel de huéspedes amplificadores vertebrados que desarrollen grandes rickettsiemias es indispensable para el mantenimiento de la rickettsia en su ciclo enzoótico, al infectar nuevas garrapatas y comenzar nuevos linajes de garrapatas infectadas (12).

Los principales huéspedes de $A$. cajennense son los caballos, el ganado, los tapires (Tapirus terrestris) y los capibaras (Hydrochoerus hydrochaeris), que son infectados por todos los estadios de $A$. cajennense (10). Los humanos también pueden ser atacados por todos los estadios de la garrapata, usualmente por cientos de larvas, docenas de ninfas y pocos adultos (4).

El capibara, también conocido en Colombia como chigüiro, pertenece al orden Rodentia, especie Hydrochoerus hydrochaeris. Es el roedor más grande en existencia, de hábito herbívoro y vive en ambientes acuáticos con diferentes combinaciones de hábitats. El capibara se considera típicamente suramericano, ya que se encuentra en las tierras cálidas desde el este del Canal de Panamá hasta el noreste de Argentina, incluyendo Colombia, las Guyanas y Uruguay (13).

En el estado de São Paulo (Brasil), un área endémica para fiebre maculosa de Brasil, los capibaras son los principales huéspedes para la garrapata $A$. cajennense. Se tienen pruebas de que el aumento de casos de fiebre maculosa del Brasil está relacionado con la presencia de capibaras en la zona, ya que son huéspedes amplificadores de Rickettsia y huéspedes primarios de la garrapata transmisora de la enfermedad (12).

Por ser vulnerables a $R$. rickettsii y por ser huéspedes primarios de $A$. cajennense, los capibaras pueden usarse como modelos centinelas para la infección por Rickettsia sp. en un área geográfica 
determinada. En Colombia son pocos los estudios de seroprevalencia en rickettsias del grupo de las fiebres manchadas en animales domésticos y silvestres; se desconoce si especies diferentes a $R$. rickettsii circulan en un ciclo enzoótico.

El objetivo de este estudio fue detectar anticuerpos contra Rickettsia sp. del grupo de las fiebres manchadas, en capibaras de una zona rural del municipio de Montería, departamento de Córdoba.

\section{Materiales y métodos}

\section{Tipo de estudio y muestreo}

Se llevó a cabo un estudio descriptivo, prospectivo y de corte transversal, para determinar la seroprevalencia de anticuerpos contra Rickettsia del grupo de las fiebres manchadas. La población la constituyeron 36 capibaras (H. hydrochaeris) de vida libre de una zona rural de la vereda San Jerónimo del municipio de Montería, departamento de Córdoba.

\section{Aspectos demográficos, climáticos y ecológicos del sitio de estudio}

La vereda San Jerónimo esta ubicada a 8`31' 20”'N y $75^{\circ} 50^{\prime} 38^{\prime \prime} O$. Contiene una reserva natural de 16 hectáreas, aproximadamente, con un humedal rodeado de vegetación riparia arbórea. El clima es cálido y húmedo tropical, con una temperatura media de $28^{\circ} \mathrm{C}$, precipitación media anual de 1.200 $\mathrm{mm}$ y dos épocas climáticas bien marcadas en el año: una época de lluvias (mayo a noviembre) donde cae el $73 \%$ de la precipitación anual, y una época seca (diciembre a abril). La fauna incluye $\mathrm{H}$. hydrochaeris, Iguana iguana, Ameiva sp., Crocodylus fuscus y varias especies de aves. La vegetación periférica de ciperáceas y gramíneas representa una importante oferta de alimento para los capibaras.

\section{Métodos serológicos}

A cada animal se le tomó una muestra de sangre por punción de la vena femoral. Las muestras se transportaron al laboratorio conservándolas a una temperatura de $4{ }^{\circ} \mathrm{C}$. Luego, se centrifugaron a $1.500 \mathrm{~g}$ por cinco minutos. El suero se conservó a $-20{ }^{\circ} \mathrm{C}$ hasta el momento del análisis. Para la detección de anticuerpos (lgG) se practicó inmunofluorescencia indirecta (IFI), con antígenos derivados de la cepa Taiaçu de $R$. rickettsii de Brasil y el conjugado anti-lgG de capibara marcado con isocianato de fluoresceína (producido por el Centro de Control de Zoonoses de São Paulo, Brasil). La preparación de antígeno y el procedimiento de la técnica se llevaron a cabo de acuerdo con el descrito por Pacheco et al. (14). Los sueros de los capibaras, o chigüiros, fueron diluidos 1:64, la serorreactividad se observó en un microscopio de fluorescencia mediante observación de color verde brillante sobre los cocobacilos extracelulares o intracelulares fijados en el portaobjeto. En cada lámina se usaron un control negativo y uno positivo (suero de chigüiro experimentalmente infectado por $R$. rickettsii y no infectado). Todas las pruebas se reconfirmaron tres veces.

A los chigüiros se les extrajo la mayor cantidad posible de garrapatas que se encontraran parasitándolos. Éstas se colocaron en tubos plásticos y se llevaron al laboratorio para su posterior clasificación (15). Cada animal capturado fue marcado para asegurarse de que sólo fuera analizado una vez.

\section{Aspectos éticos}

El estudio fue aprobado por el Comité de Ética del Instituto de Investigaciones Biológicas del Trópico, adscrito a la Facultad de Medicina Veterinaria de la Universidad de Córdoba. La aprobacion del proyecto garantizó que los animales no sufrieran durante la obtención de las muestras y no se hizo ningún procedimiento que pusiera en riesgo la salud de los animales. Para la obtención de las muestras, se administró previamente anestesia al animal bajo estricta vigilancia de un médico veterinario. No se muestrearon animales que, por su tamaño, senectud o estado de salud, pusieran su vida en peligro. Las muestran se obtuvieron según las condiciones del sitio y manteniendo las normas de bioseguridad establecidas para tal fin. Se tuvo en cuenta la Resolución No. 008430 de 1993 (4 de octubre de 1993) del Ministerio de Salud de la República de Colombia "[...] por la cual se establecen las normas académicas, técnicas y administrativas para la investigación en salud, concernientes a la investigación biomédica con animales", artículo 87, literales c, g y h.

Los investigadores de este estudio conocían el Decreto 309 de 2000 del Ministerio del Medio Ambiente en la República de Colombia "[...] por el cual se reglamenta la investigación científica sobre la diversidad biológica", artículo $2^{\circ}$, parágrafo $1^{\circ}$. Y la "Declaración universal de los derechos de los animales" proclamada por la Liga Internacional de los Derechos del Animal (UNESCO, 1989, Ginebra, Suiza), la "Guía para el cuidado y uso de animales de laboratorio" (NRC, 1996) y los "Principios éticos de la experimentación animal" enunciados por el 
Internacional Council for Laboratory Animal Science (ICLAS) (http://dels.nas.edu/ilar_n/ilarhome/).

\section{Resultados}

Entre mayo y junio de 2009, se capturaron 36 capibaras, de los cuales, 10 (28\%) eran machos y 26 (72\%), hembras. De estos animales, cuatro eran machos adultos, 6 machos juveniles, 17 hembras adultas, 2 hembras subadultas y 7 hembras juveniles.

De los 36 sueros analizados por IFI, el $22 \%$ (8 capibaras) presentó anticuerpos contra Rickettsia sp. del grupo de la fiebres manchadas. Se encontraron cuatro sueros con título de 1:64, tres sueros con título de 1:128 y un suero presentó titulación de 1:512. En el cuadro 1 se resumen las principales características de los capibaras que resultaron positivos.

Se recolectaron 933 garrapatas, $658(70,5 \%)$ machos, 160 (17,1\%) hembras, 105 (11,3\%) ninfas y $4(0,43 \%)$ larvas. Todas las garrapatas adultas extraídas de los chigüiros se identificaron taxonómicamente como $A$. cajennense. Las ninfas y larvas se consideraron que correspondían morfológicamente a $A$. cajennense.

\section{Discusión}

Colombia es una zona endémica de rickettsiosis. La enfermedad en el país fue descrita en 1937 por Patiño et al. en la localidad de Tobia (Cundinamarca) (16) y no se volvió a reportar hasta el año 2001, cuando se realizó un estudio de seroprevalencia en el municipio de Ciénaga de Oro (Córdoba) donde se reportó una seroprevalencia de $49 \%$ (17). Dos años después, se estudiaron dos casos mortales de rickettsiosis en una comunidad cercana a Villeta y Tobia (Cundinamarca) (18). Recientemente, en los departamentos de Córdoba y Antioquia, se han presentado brotes esporádicos de rickettsiosis con altas tasas de letalidad: Necoclí en el 2006 (19), Los Córdobas en el 2007 (20) y en Altos de Mulatos en el 2008 (21). Por lo general, donde se presentaron los brotes fueron áreas rurales, donde abundan los animales domésticos y donde fue descrita la presencia de la garrapata $A$. cajennense (18-21).

En nuestro estudio, todas las garrapatas encontradas parasitando a los capibaras se identificaron como A. cajennense. En Brasil, donde los principales huéspedes de $A$. cajennense son los caballos y los capibaras, el aumento de la población de estos últimos se ha relacionado con el incremento de casos de fiebre manchada de Brasil en áreas endémicas para esta rickettsiosis (12).

Brasil reporta las mayores tasas de rickettsiosis en Suramérica, con 350 casos confirmados por laboratorio en la región del sureste del país entre 1995 y 2004 y 128 casos confirmados entre 2005 y 2007 en Sao Paulo. En este país es donde mejor se ha estudiado la ecología de las rickettsias (4). Los estudios hechos en animales centinelas para la vigilancia de las rickettsias, como el de Horta et al., en el área de Pedreira (São Paulo, Brasil), un área endémica de rickettsiosis, determinó $77,3 \%$ y $31 \%$ de seroprevalencia de $R$. rickettsii en caballos y perros, respectivamente (22).

En Colombia, a pesar de tener zonas endémicas de rickettsiosis, son pocos los estudios realizados en animales como modelos centinela para determinar la presencia de especies de rickettsias en el área. Hidalgo et al., en un trabajo llevado a cabo en una zona endémica del país, encontraron una seropositividad para Rickettsia spp. en caballos de $16,3 \%$ y de $18,2 \%$ en caninos (23). Nuestro trabajo es el primero realizado en Colombia en el que se utilizan a los capibaras $(H$. hydrochaeris) como un

Cuadro 1. Garrapatas y titulo final de la inmunofluorescencia indirecta para Rickettsia rickettsii en chigüiros de la vereda San Jerónimo del municipio de Montería, Córdoba.

\begin{tabular}{lllrl}
\hline Animal & $\begin{array}{c}\text { Estado } \\
\text { fisiológico }\end{array}$ & Sexo & $\begin{array}{c}\text { IFI, título final } \\
\text { Número de garrapatas } \\
\text { y estadio }\end{array}$ \\
\hline 2 & Juvenil & Macho & 128 & $30 \mathrm{M}, 8 \mathrm{H}$, \\
13 & Adulto & Hembra & 64 & $17 \mathrm{M}, 5 \mathrm{H}, 9 \mathrm{~N}$ \\
15 & Adulto & Hembra & 128 & $11 \mathrm{M}, 6 \mathrm{H}, 2 \mathrm{~N}$ \\
17 & Adulto & Hembra & 512 & $8 \mathrm{M}$, \\
18 & Juvenil & Hembra & 64 & $8 \mathrm{M}, 3 \mathrm{H}$, \\
19 & Adulto & macho & 64 & $10 \mathrm{M}, 12 \mathrm{H}$, \\
25 & Adulto & Hembra & 64 & $10 \mathrm{M}$, \\
34 & Juvenil & Macho & 128 & $31 \mathrm{M}, 1 \mathrm{H}, 3 \mathrm{~N}$ \\
\hline
\end{tabular}

M: macho; H: hembra; N: ninfa

IFI: inmunofluorescencia indirecta 
posible centinela para detectar la circulación de rickettsias del grupo de las fiebres manchadas.

En los estudios de Souza et al. (12), se demostró que los chigüiros son propensos a la infección por $R$. rickettsii y tienden a desarrollar un periodo de rickettsiemia de alrededor de 10 días consecutivos (7 a 12 días), sin presentar signos de fiebre. En contraste, en otras especies de mamíferos (humanos, perros, cobayos y conejos domésticos), $R$. rickettsii induce fiebres altas e incluso la muerte. Los capibaras, al desarrollar altas y prolongadas rickettsiemias, podrían jugar un papel importante en la diseminación de las rickettsias, ya que se ha demostrado que al ser picados por garrapatas no infectadas éstas adquieren la infección (12).

En un estudio de Souza et al., en áreas endémicas de rickettsiosis en São Paulo (Brasil), se reportó 7,3 \%, $37,5 \%, 41,6 \%, 53,3 \%$ y $59,4 \%$ se seroprevalencia en capibaras, en cinco localidades de la región de Las Campinas, para una seroprevalencia total de 32,3\% (57/177); el antígeno usado fue $R$. rickettsii (24).

En nuestro estudio la seroprevalencia encontrada fue de $22 \%(8 / 36)$ y, al igual que en el estudio anterior, el antígeno fue $R$. rickettsii. Aunque en ambos estudios el antígeno usado fue el mismo, es importante indicar que la inmunofluorescencia indirecta, a pesar de tener una alta sensibilidad para $R$. rickettsii, no discrimina el agente etiológico debido a la reacción cruzada que presenta en el grupo de la fiebres manchadas. En Colombia, se desconoce si existen otras especies de rickettsias del grupo de las fiebres manchadas diferentes a $R$. rickettsii y no se puede descartar la posibilidad de que existan éstas o una nueva especie.

En otro estudio en Brasil, en áreas no endémicas (14), se evaluaron los sueros de capibaras con todas las especies de rickettsias encontradas en el estado de São Paulo y se reportó una seroprevalencia de 74\% (54/73). La seroprevalencia para cada especie fue de 19 (26,0\%), 25 (34,2 $\%)$ y $50(68,5 \%)$ sueros que reaccionaron con $R$. rickettsii, R. parkeri y $R$. bellii, respectivamente. Sin embargo, debido a la reacción cruzada entre estos microorganismos, se determinó realmente que 25 sueros fueron homólogos a $R$. bellii, 3 sueros lo fueron a $R$. parkeri y ningún suero fue homólogo a $R$. rickettsii. En este estudio se reportaron por primera vez capibaras infectados naturalmente por $R$. bellii y $R$. parkeri. La evaluación de sueros contra la especie de rickettsia conocida en un área determinada es ideal, porque frecuentemente los títulos de anticuerpos homólogos son más altos que los títulos de anticuerpos heterólogos. En algunos casos, la diferencia en títulos puede ser suficiente para diferenciar entre las especies de rickettsia que estimulan el sistema inmunitario (25).

El presente estudio reporta por primera vez la infección natural por una rickettsia del grupo de las fiebres manchadas, en capibaras de Colombia. La seroprevalencia encontrada pudo haber estado afectada por el reducido tamaño de muestras analizadas (36 capibaras), a diferencia de los estudios en Brasil $(14,24)$. Sería importante ampliar el estudio de seroprevalencia a la población humana y a animales domésticos del área estudiada, ya que están en estrecho contacto con los capibaras.

Por otro lado, debido a la presencia de reacción cruzada entre el grupo de las fiebres manchadas, los resultados deben interpretarse cuidadosamente, y se requiere de métodos capaces de distinguir entre las especies de rickettsias. Nuestros hallazgos sugieren que los capibaras pueden ser usados como potenciales centinelas de la circulación de rickettsias y marcadores de las áreas de riesgo para la transmisión de rickettsiosis.

\section{Agradecimientos}

A Santiago Monsalve, por la toma de muestras en los chigüiros, y al personal que labora en la reserva natural de la vereda San Jerónimo.

\section{Conflictos de intereses}

El manuscrito fue preparado y revisado con la participación de todos los autores, quienes declaramos que no existe ningún conflicto de intereses que ponga en riesgo la validez de los resultados.

\section{Financiación}

Este estudio fue financiado por la Facultad de Medicina Veterinaria y Zootecnia de la Universidad de Córdoba.

\section{Referencias}

1. Wölfel R, Essbauer S, Dobler G. Diagnostics of tick-borne rickettsioses in Germany: A modern concept for a neglected disease. Int J Med Microbiol. 2008;298:368-74.

2. Weinert LA, Werren JH, Aebi A, Stone GN, Jiggins FM. Evolution and diversity of Rickettsia bacteria. BMC Biol. 2009;7:6.

3. Gillespie JJ, Beier MS, Rahman MS, Ammerman NC, Shallom JM, Purkayastha A, et al. Plasmids and rickettsial evolution: Insight from Rickettsia felis. PLoS One. 2007;2:e266. 
4. Labruna MB. Ecology of Rickettsia in South America. Ann N Y Acad Sci. 2009;1166:156-66.

5. Pacheco RC, Moraes-Filho J, Nava S, Brandão PE, Richtzenhain LJ, Labruna MB. Detection of a novel spotted fever group rickettsia in Amblyomma parvum ticks (Acari: Ixodidae) from Argentina. Exp Appl Acarol. 2007;43:63-71.

6. Paddock CD, Fournier PE, Sumner JW, Goddard J, Elshenawy Y, Metcalfe MG, et al. Isolation of Rickettsia parkeri and identification of a novel spotted fever group Rickettsia sp. from Gulf Coast ticks (Amblyomma maculatum) in the United States. Appl Environ Microbiol. 2010;76:268996.

7. Parola P, Raoult D. Tropical rickettsioses. Clin Dermatol. 2006;24:191-200.

8. Guedes E, Leite RC, Prata MC, Pacheco RC, Walker DH, Labruna MB. Detection of Rickettsia rickettsii in the tick Amblyomma cajennense in a new Brazilian spotted fever-endemic area in the state of Minas Gerais. Mem Inst Oswaldo Cruz. 2005;100:841-5.

9. Sangioni LA, Horta MC, Vianna MC, Gennari SM, Soares RM, Galvão MA. Rickettsial infection in animals and Brazilian spotted fever endemicity. Emerg Infect Dis. 2005;11:265-70.

10. Labruna MB, Whitworth T, Horta MC, Bouyer DH, McBride JW, Pinter A, et al. Rickettsia species infecting Amblyomma cooperi ticks from an area in the state of Sao Paulo, Brazil, where Brazilian spotted fever is endemic. Clin Microbiol. 2004;42:90-8.

11. Labruna MB, Ogrzewalska M, Martins TF, Pinter A, Horta MC. Comparative susceptibility of larval stages of Amblyomma aureolatum, Amblyomma cajennense, and Rhipicephalus sanguineus to infection by Rickettsia rickettsii. J Med Entomol. 2008;45:1156-9.

12. Souza CE, Moraes-Filho J, Ogrzewalska M, Uchoa FC, Horta MC, Souza SS, et al. Experimental infection of capybaras Hydrochoerus hydrochaeris by Rickettsia rickettsi and evaluation of the transmission of the infection to ticks Amblyomma cajennense. Vet Parasitol. 2009;161:11621.

13. Ojasti J. Utilización de fauna silvestre en América Latina: situación y perspectivas para un manejo sostenible. Guía FAO de conservación 25. Fecha de consulta: 22 mayo de 2010. Disponible en: http://www.fao.org/docrep/006/t0750s/ t0750s00.html.

14. Pacheco RC, Horta MC, Moraes-Filho J, Ataliba AC, Pinter A, Labruna MB. Rickettsial infection in capybaras (Hydrochoerus hydrochaeris) from São Paulo, Brazil: Serological evidence for infection by Rickettsia bellii and Rickettsia parkeri. Biomédica. 2007;27:364-71.
15. Onofrio V, Labruna M, Pinter A, Giacomin F, BarrosBattesti D. Comentarios e chaves para as espécies do genero Amblyomma. In: Barros-Battesti D, Arzua M, Bechara G. Carrapatos de importancia medico veterinaria da regiao neotropical. Um guía ilustrado para identificaçao de espécies. Sao Paulo: Butantan; 2006. p. 53-113.

16. Patiño L, Afanador A, Paul JH. A spotted fever in Tobia, Colombia. Am J Trop Med Hyg. 1937;17:639-53.

17. Miranda A, Florez S, Máttar S. Alta seroprevalencia de rickettsiosis en trabajadores del campo en el municipio de Ciénaga de Oro, Córdoba. Inf Quinc Epidemiol Nac. 2001;7:71-5

18. Hidalgo M, Orejuela L, Fuya P, Carrillo P, Hernández J, Parra E, et al. Rocky Mountain spotted fever, Colombia. Emerg Infect Dis. 2007;13:1058-60.

19. Acosta J, Urquijo L, Díaz A, Sepúlveda M, Mantilla G, Heredia D, et al. Brote de rickettsiosis en Necoclí, Antioquia, febrero -marzo de 2006. Inf Quinc Epidemiol Nac. 2006;11:177-92

20. Hidalgo M, Miranda J, Heredia D, Zambrano P, Vesga JF, Lizarazo D, et al. Outbreak of Rocky Mountain spotted fever in Córdoba, Colombia. Mem Inst Oswaldo Cruz. 2011;106:117-8.

21 Pacheco O, Giraldo M, Hidalgo M, Galeano A, Echeverri I, Echevarria L, et al. Estudio de brote febril hemorrágico en el corregimiento de Alto de Mulatos - Distrito Especial Portuario de Turbo, Antioquia, enero de 2008. Inf Quinc Epidemiol Nac. 2008;13:145-60.

22. Horta MC, Labruna MB, Sangioni LA, Vianna MC, Gennari SM, Galvão MA, et al. Prevalence of antibodies to spotted fever group rickettsiae in humans and domestic animals in a Brazilian spotted fever-endemic area in the state of Sao Paulo, Brazil: Serologic evidence for infection by Rickettsia rickettsii and another spotted fever group Rickettsia. Am J Trop Med Hyg. 2004;71:93-7.

23. Hidalgo M, Vesga JF, Lizarazo D, Valbuena G. A survey of antibodies against Rickettsia rickettsii and Ehrlichia chafeensis in domestic animals from a rural area of Colombia. Am J Trop Med Hyg. 2009;80:1029-30.

24. Souza CE, Lacerra SS, Castor VL, Berger S, Gibrail M, Mouriz E, et al. Serological identification of Rickettsia spp. from the spotted fever group in capybaras in the region of Campinas - SP - Brazil. Ciencia Rural. 2008;38:1694-9.

25. La Scola B, Raoult D. Laboratory diagnosis of rickettsioses: Current approaches to diagnosis of old and new rickettsial diseases. J Clin Microbiol. 1997;35:2715-27. 